\title{
On the relationship between neck length and bond radius during compression of snow
}

\author{
R. L. BRown AND M. Q. Edens \\ Civil and Applied Engineering Department, Montana State University, Bozeman, Montana 59715, U.S.A.
}

\begin{abstract}
In an earlier study on the variations in micro-structure during large volumetric deformations of snow, the authors observed that, contrary to expectations, the length of necked regions connecting adjacent grains did not necessarily decrease during compression. Rather, there was no discernible or predictable change in neck length, in some cases increasing and in others decreasing. Further evaluations of the data and an analysis of the mechanics of neck deformation determined that the process is complicated by three different effects: (1) increase in coordination number (number of bonds per grain), (ii) plastic deformation of the neck, and (iii) a geometric effect determined by bond growth and grain geometry. It is found that the first two effects tend to decrease the neck length and that the third produces an increase in mean neck length. A set of coupled differential equations is developed describing the variation of neck length and bond radius, and solved numerically for conditions consistent with the experimental data. Calculated results agree well with the data for the bond radius but the results for the neck length are less satisfactory. Reasons for this lie with difficulty in making accurate measurements of mean neck length from two-dimensional surface-section data and in the criteria for the definition of necks.
\end{abstract}

\section{INTRODUCTION}

Micro-structural properties are important for determining the physical and mechanical properties of any material. In the case of granular materials such as snow, the geometry of the grains and the connecting units, the necks which tie the material together, are among these micro-structural properties (Kry, 1975a, b; Gubler, 1987a, b; Brown, 1980). Necks are defined as those constricted areas joining two ice grains. The neck begins where the surface curvature changes from convex outward to concave outward as one moves on the ice surface from the grain toward the neck. Figure 1 illustrates a neck, though this example shows a neck which is considerably longer than is normally found in alpine snow. The bonds are usually defined to be the minimum cross-sectional area on the necks. In non-cohesive materials such as sand, necks do not develop, and grainto-grain contact is accomplished by simple contact areas which are also referred to as bonds. These bonds can transmit shear and compressive normal forces, with the shear forces governed by a friction law such as the MohrCoulomb law.

In cohesive materials such as snow, a number of processes are responsible for the development of a necked region of finite length and cross-sectional area joining the contacting particles together. The neck growth results

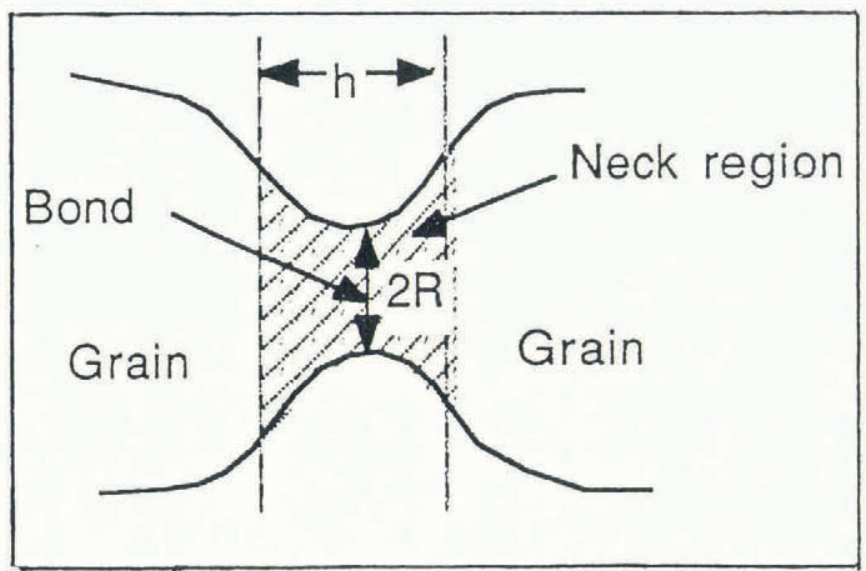

Fig. 1. Schematic of neck and bond as they appear in a surface section.

because of the combined effects of pressure sintering, vapor diffusion, volume diffusion and grain-boundary diffusion (Maeno and Ebinuma, 1983). Usually, for a given set of conditions, one or two processes will dominate. For instance, under normal conditions for alpine snowpressure sintering and vapor diffusion can be expected to be the two dominant processes for the formation and growth of necks. 
Necks are particularly easy to detect in snow that has gone through a melt/freeze process, since during the time snow contains free water on the grain surfaces, the water collects at the necks and upon freezing forms large necks that are easy to detect. In the case of dry alpine snow, the highly irregular grain shape and relatively small dimensions of the necked regions make the measurement of the neck size a more subjective and difficult process.

What has not been fully appreciated in the past has been the importance of necks in determining behavior of snow and, for that matter, some physical properties as well. In normal alpine snow with a density $\rho$ of approximately $250 \mathrm{~kg} \mathrm{~m}^{-3}$, neck lengths can be on the order of $10 \%$ of the grain diameter, while the neck (or bond) diameter may be $20 \%$ of the grain diameter. Under these conditions, the necks do not have a predominant effect on the value of Young's modulus for the material, since a linear elastic constitutive behavior for the ice is assumed in calculating the elastic modulus. However, when considering long-term slow plastic deformation of snow, a constitutive behavior analogous to Glen's flow law must be used for the ice phase. In this case, the deformation in the necks is responsible for much of the snow deformation, since the necks are subjected to stresses almost two orders of magnitude higher than the bulk snow stress. Since the plastic strain rate for ice varies with the cube of the applied stress, one can easily verify that neck deformations account for much of the observed deformation in the snow. This picture is complicated somewhat when rates are large enough to cause significant bond fracture with resultant intergrannular slip, but the strength properties and geometry of the necks still play a significant role in determining the frature processes in the bonds.

In an earlier paper (Edens and Brown, 1991), an image-analysis technique based on quantitative stereology (Underwood, 1970) and developed by Hansen and Brown (1986) was used to evaluate the variation of

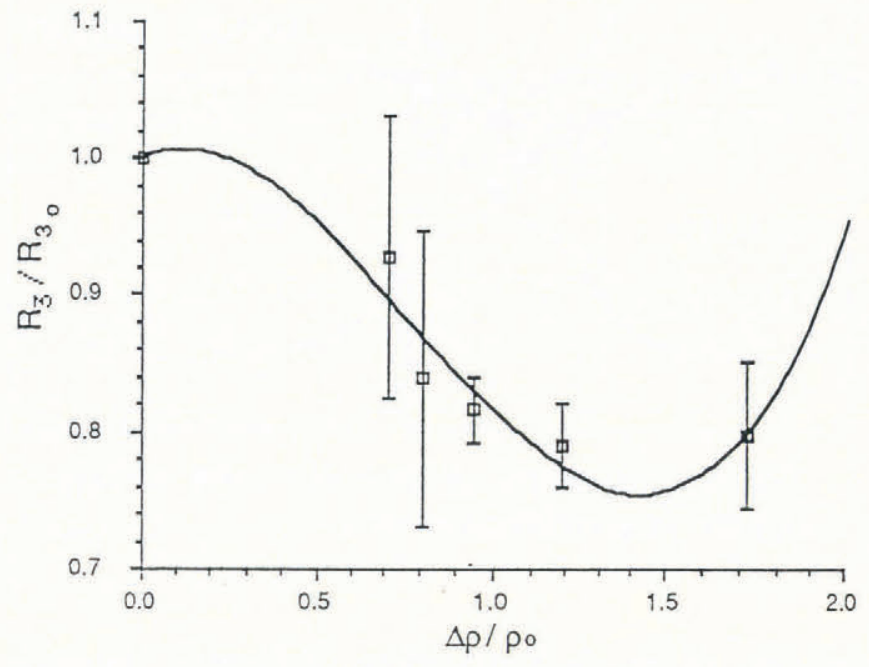

Fig. 2. Relationship between ratio of threedimensional bond radius to initial threedimensional bond radius and density change divided by initial density. The vertical lines represent the total range of the data scatter. The cubic least-squares fit represented by the curve had a standard error $S_{R}=0.025$.

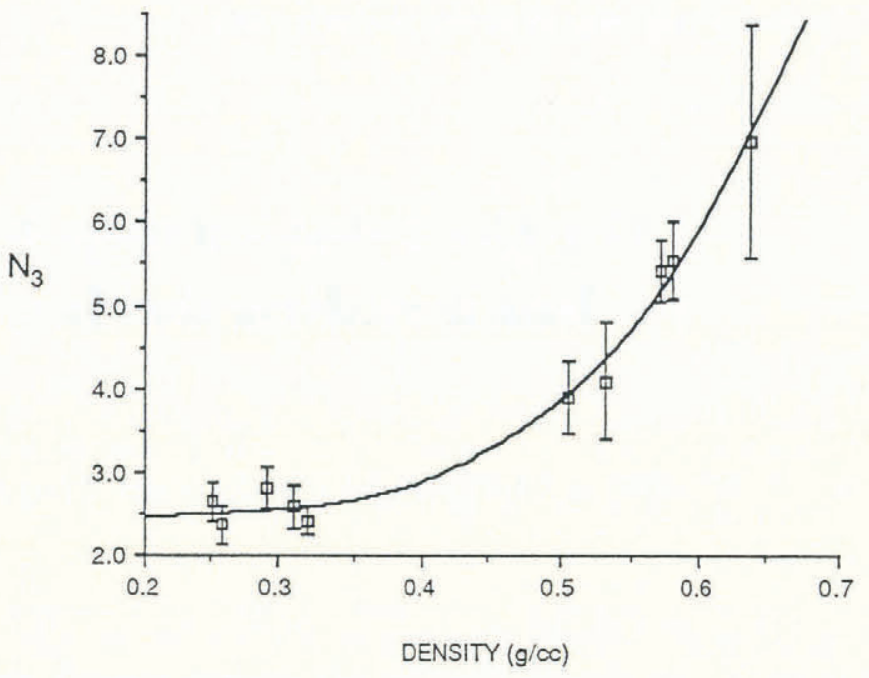

Fig. 3. Three-dimensional coordination number, $N_{3}$, vs density. The vertical lines represent the total range of the data scatter. The curve is a cubic least-squares fit to the data with a standard error of $S_{N}=0.120$.

micro-structure during large compressive deformations of snow samples. Surface sections were made from test samples at various stages of deformation and analyzed with an image-analysis system to determine changes in the three-dimensional micro-structural properties of the material. Typical results are shown in Figures 2 and 3 which demonstrate, respectively, the variation of mean bond radius $R_{3}$ and three-dimensional coordination number $N_{3}$ with deformation as snow samples were subjected to confined compression from initial densities of about 300 to approximatley $600 \mathrm{~kg} \mathrm{~m}^{-3}$. The three-dimensional coordination number, $N_{3}$, is defined as the number of bonds per grain. As can be seen in Figure 2, the mean bond radius goes through a fairly complicated variation, first changing very little, then decreasing markedly, and eventually levelling off. This behavior was attributed to the formation of new bonds during compression. Since these new bonds would necessarily be very small at the time of initial contact between adjoining grains, the effect would decrease the average bond size in the material. In Figures 2 and 3 one can note that $R_{3}$ does not begin to decrease significantly until $N_{3}$ begins to undergo large increases in value.

The coordination number went through a predictable increase in value, at first not changing substantially until pore size was reduced enough to begin increasing the rate of new contact between grains (Fig. 3). The reader is referred to the previous papers by the authors for a detailed description of the analysis and results. The curves in these figures are least-square fits of a cubic equation to the data. The vertical bars represent the total range of the data scatter of the variable values (in this case $R_{3}$ and $N_{3}$ ) evaluated in the image analysis. Two or three different surface sections were taken for each test sample and the calculated variables were subsequently averaged to obtain the results shown in these figures.

One difficulty encountered in the analysis by Edens and Brown (1991) was the inability to obtain predictable change patterns in the neck length, $h$, during compres- 


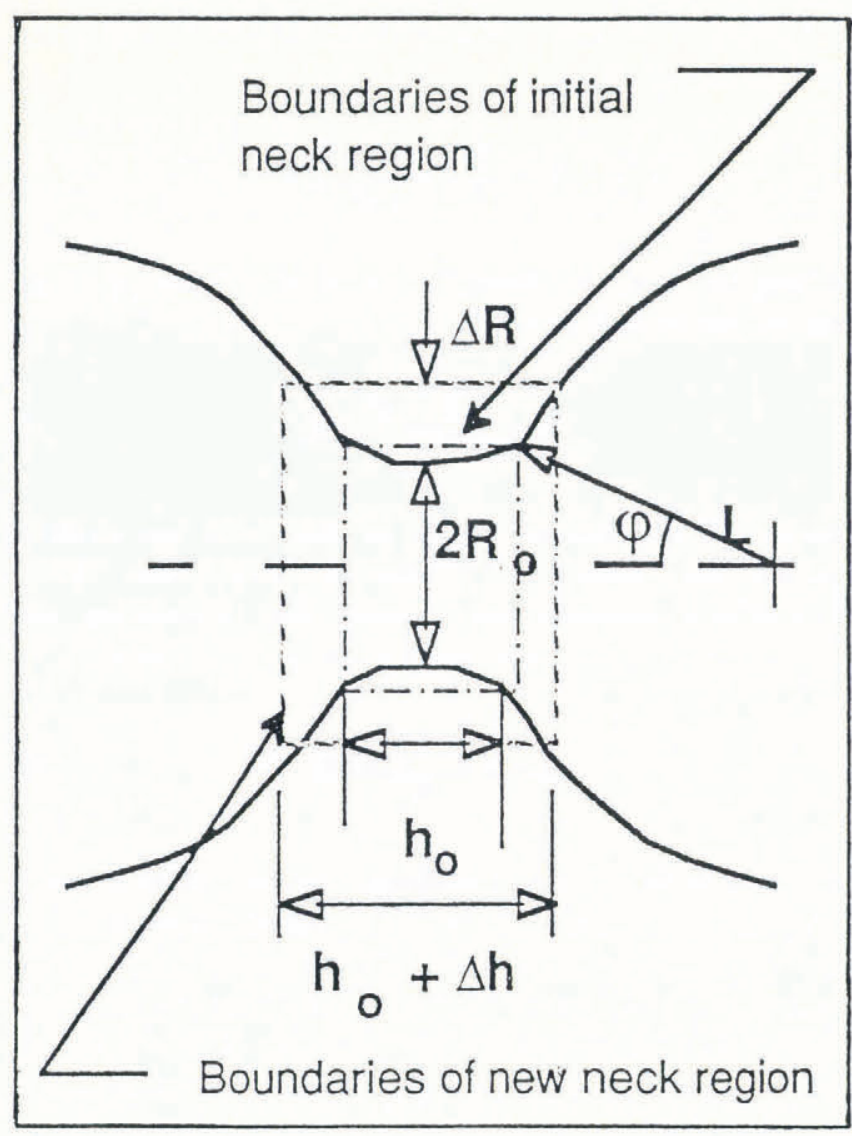

Fig. 4. Schematic of the geometric effect which produces a change in neck length due to an increase in bond radius.

sive deformation. In some cases the mean neck length actually increased during deformation and in other cases it showed the expected decrease in value. There were some possible explanations for this surprising result. One could be that in spite of pressure sintering and the formation of new bonds during compression, neck length could increase due to bond growth, causing the neck actually to grow into the grain as the necks expand laterally. This effect is illustrated in Figure 4. As the necks grow laterally (i.e. as $R_{3}$ increases) due to sintering, the neck will extend further on to the surface of the grain. Therefore, if the neck radius increases by the amount $\Delta R_{3}$, the neck will increase by the amount $\Delta h$ indicated in Figure 4. In addition to the change in $\Delta R_{3}, \Delta h$ also depends on the grain-surface geometry at the junction of the neck and grain. This is readily determined if the grains are all spherical and of equal size, but in the case of alpine snow the irregular shape of the particles precludes a precise determination of this geometry. This one process is not expected to be significant enough to offset the effects of sintering and new bond formation, both of which produce decreases in mean neck length. However, this needed to be studied in a more rigorous manner. In the following an analysis is made of the deformation of the necked regions to determine in detail just how the mean bond radius and neck length change during volumetric compression of snow.

\section{ANALYSIS}

A number of different processes are responsible for changes in neck length and bond radius. Consider first the changes in neck length. These include (i) the effect of increasing coordination number $N_{3}$ (bonds per grain) during which new bonds are formed with very small initial bond radius, (ii) compressive axial plastic deformations in the necks, and (iii) a geometric effect due to the neck growing into the grains as the bond radius increases.

Figure 4 illustrates the geometric effect associated with an increase in bond radius resulting from bond growth due to pressure sintering and other effects. The ice grain is assumed to have a radius of curvature $L$ at the point where the neck joins to the grain. The subscript " 3 " is used to indicate that the variables being discussed are three-dimensional variables, such as $N_{3}, R_{3}$ and $h_{3}$. In what follows, however, this subscript is dropped in order to simplify the ensuing equations. It should be remembered that we are dealing with three-dimensional variables rather than surface-section variables.

Assume that over a period of time $\Delta t$ the neck radius $R$ grows by the amount $\Delta R$. If the instantaneous neck length is $h$, the growth of neck length is found to be:

$$
\Delta h / \Delta R=\tan \varphi .
$$

$\varphi$ is the slope of the grain surface at the grain/neck junction. If the grain is assumed to be spherical, then $\varphi$ also represents the angle shown in Figure 4. However, for the purposes of this study, such an assumption is not made.

Dividing both $\Delta R$ and $\Delta h$ by a time increment $\Delta t$ and taking $\Delta t$ to zero in the limit, the following results:

$$
\dot{h}=\dot{R} \tan \varphi
$$

If $\varphi$ is small, the tangent may be replaced by the term $\sin (\varphi)$, which has the value $R / L$. However, the slope of

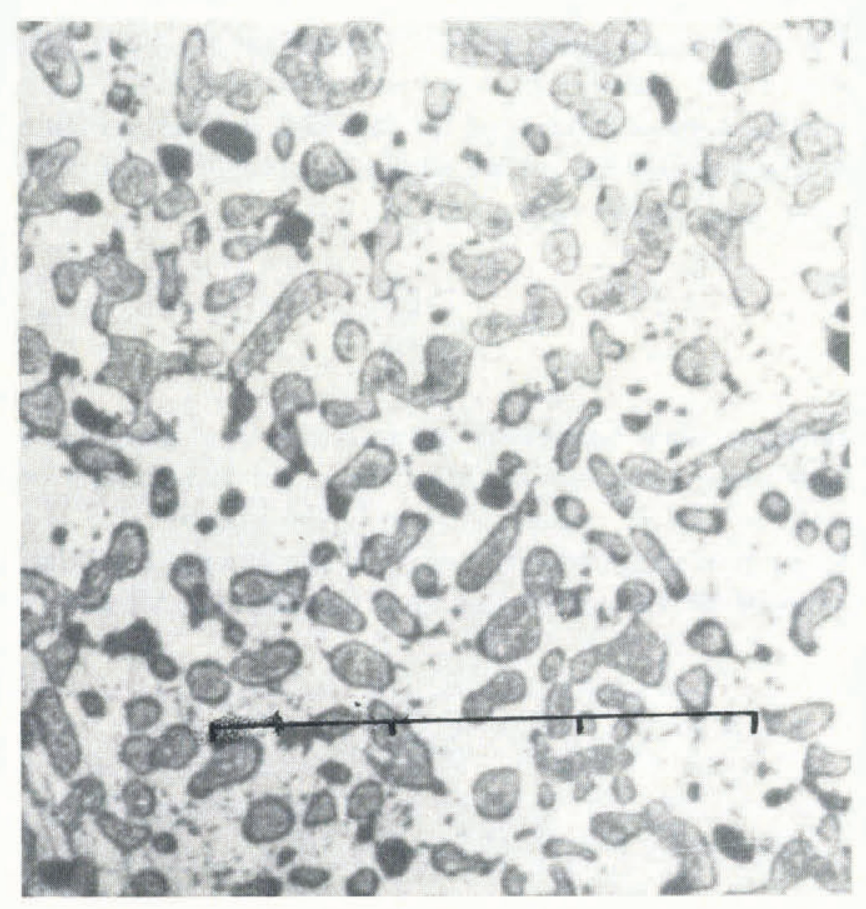

Fig. 5. A typical surface section of snow samples used in this study. Note that this snow has no faceting, indicating long-term storage under uniform temperature conditions. 
the grain surface at the junction of the grain and neck, as given by $\tan \varphi$, has a strong influence on the value of $\mathrm{d} h / \mathrm{d} t$. The spherical grain geometry used in developing Equation (2) is a poor approximation of the actual grain geometry in alpine snow. Figure 5 illustrates a surface section of snow which is typical of what was used in the study by Edens and Brown (1991). This snow displays the effects of long-term equilibrium metamorphism which produces the rounded grains and substantial intergranular bonding. Investigation of a number of surface sections indicated that the term $\tan \varphi$ overestimates the grain-surface slope at the neck by a substantial factor, particularly for small necks. As a consequence, in the calculations which follow, the term $\tan \varphi$ is replaced by the term $C \cdot R / L$, where $C$ is a constant. $R / L$ approximates the $\tan \varphi$ and reflects the role that the ratio of the bond and grain radii play in determining the rate of growth of the neck length. The rate of change of $h$ described in Equation (2) is referred to as the "geometric effect", since it is determined by the neck and grain geometry.

As can be seen in Figure 4 and Equation (2), the geometric effect results from the growth of the bond radius $R$. As $R$ increases, more of the grain free surface is consumed by the neck itself, and the effective length of the neck is increased. How mass is added to increase the radius $R$ is irrelevent for this effect. It could be due to plastic deformation in the neck, vapor diffusion to the neck, volume or surface-diffusion effects or melt/freeze processes.

Both $R$ and $h$ are affected by the additions of new contacts between grains during densification. These will normally result with decreases in mean values of $R$ and $h$. When a new contact is made between two grains, the radius of the new contact will be small, but not necessarily zero. The bond radii of new contacts depend on the geometry of the contacting surfaces, so the mean value of a new bond radius will be denoted by $R_{0}$. The new neck is also assumed to have an initial length $h_{0}$. Now consider some volume $V$ of the material, and assume this volume contains $M$ grains with a total of $N$ necks. The mean bond radius $R$ is given by:

$$
R=\frac{1}{N} \sum_{i=1}^{N} R_{i}
$$

During a time increment $\Delta t$ in which the material is undergoing compressive deformation, let the number of bonds in the $M$ grains increase to the number $N^{\prime}$. For the $M$ grains considered here, the resulting mean bond radius $R^{\prime}$ at the end of this time increment is then:

$$
R^{\prime}=\frac{1}{N^{\prime}} \sum_{i=1}^{N^{\prime}} R_{i}=\frac{1}{N^{\prime}}\left[\sum_{1}^{N} R_{i}+\sum_{N+1}^{N^{\prime}} R_{i}\right]
$$

This can be re-arranged to the form:

$$
R^{\prime}=\frac{N}{N^{\prime}} R+\frac{R_{0}}{N^{\prime}}\left(N^{\prime}-N\right)
$$

where, again $R_{0}$ is the initial neck radius formed by a new contact.
Letting $R^{\prime}=R+\Delta R$ and $N^{\prime}=N+\Delta N$, Equation (5) can be expressed as:

$$
\Delta R=\frac{\left(R_{0}-R\right)}{(N+\Delta N)} \Delta N
$$

Taking the time increment to zero, the above equation can be put in the form:

$$
\dot{R}=\frac{\left(R_{0}-R\right)}{N_{3}} \dot{N}_{3}
$$

where the number of bonds $N$ has been normalized against the number of grains to obtain the threedimensional coordination number $N_{3}$. Following the same procedure, one can obtain for the neck length:

$$
\dot{h}=-\frac{\left(h-h_{0}\right)}{N_{3}} \dot{N}_{3}
$$

where $h_{0}$ is the initial neck length when initial contact between two grains is made. We would ordinarily take $h_{0}=0$, but the resolution of the image-analysis system used in the analysis as $0.033 \mathrm{~mm} / \mathrm{pixel}$ for the magnifications used for the stereology studies. It was assumed that a pixel would have to show before a contact point was taken to be a neck. Therefore $h_{0}$ was assumed to be $0.033 \mathrm{~mm}$ as an initial length when a new neck was registered by the image-analysis system used for this study. For the same reason, the value of $0.033 \mathrm{~mm}$ was used for the initial bond radius due to formation of new contacts.

Finally, the neck length will decrease as a result of plastic strain due to the compressive stresses applied to the neck area. These stresses are very difficult to estimate in an exact manner, so an approximate value is used. From quantitative stereology, the mean ice stress in the material is related to the bulk snow stress through the density ratio $\alpha=\rho_{\mathrm{i}} / \rho_{\mathrm{s}}$, where $\rho_{\mathrm{i}}$ and $\rho_{\mathrm{s}}$ are respectively the ice density and snow density. The stress in the necks is further augmented by the ratio of the grain crosssectional area and the neck cross-sectional area. This then gives an augmentation factor of:

$$
\frac{\sigma_{\mathrm{n}}}{\sigma_{\mathrm{s}}}=\frac{\alpha}{2 N_{3}}\left(\frac{L}{R}\right)^{2}
$$

where $\sigma_{\mathrm{n}}$ and $\sigma_{\mathrm{s}}$ are respectively the neck stress and the bulk snow stress. $L$ is the mean grain radius. Assuming that the ice obeys Glen's flow law under steady-state conditions, the axial strain rate, $\mathrm{d} \epsilon / \mathrm{d} t$, in the neck is approximated by the relation:

$$
\dot{\epsilon}=A \sigma_{\mathrm{n}}{ }^{3} \text {. }
$$

The rate of change of neck length due to this effect is then just the strain rate multiplied by the instantaneous neck length. The lateral strain in the neck is calculated by assuming that the plastic deformation is isochoric with a Poisson's ratio of $\nu=0.5$. Then the total rate of change of the mean neck length, $\mathrm{d} h / \mathrm{d} t$, is found by summing the separate effects of Equations (2), (8) and (10). This 
leads to:

$$
\dot{h}=h A\left[\sigma_{\mathrm{s}} \alpha(L / R)^{2} \frac{1}{2 N_{3}}\right]^{3}-\frac{\left(h-h_{0}\right) \dot{N}_{3}}{N_{3}}+C R \frac{\dot{R}}{L} .
$$

In Equations (9)-(12), the normal stresses are assumed to be positive in tension. Again, the term $\tan \varphi$ in Equation (2) has been replaced by the expression $C R / L$. The total rate of change of bond radius is the combined effect of Equation (7) and a Poissonic effect which can be found using Equation (10). This results with:

$$
\dot{R}=-\nu R A\left[\sigma_{\mathrm{s}} \alpha\left(\frac{L}{R}\right)^{2} \frac{1}{2 N_{3}}\right]^{3}+\frac{\left(R_{0}-r\right)}{N_{3}} \dot{N}_{3} .
$$

In order to solve these two equations for changes in mean neck length and mean bond radius, the variation of the bulk snow stress and three-dimensional coordination number must be prescribed. The coordination number $N_{3}$ is assumed to vary as indicated in Figure 2, which illustrates the coordination numbers found by using the analysis discussed by Edens and Brown (1991). As can be seen in the figure, the data have sufficient repeatability to be used with confidence in the analysis. The actual stress data obtained during the testing were used to provide values of stress during the numerical integration of these equations, so that the only unknown variables in these two equations are the neck length $h$ and the bond radius $R$. The actual stress data showed an irregular behavior during the compression tests, displaying the familiar sawtooth behavior in the stress-time curve. Rather than using this curve directly, a smoothed curve obtained with a least-squares fit was used to approximate the temporal variation of the stress during loading. Using the numerical data for the coordination number and the bulk snow stress, one can then numerically integrate Equations (11) and (12) to calculate the predicted variation of the bond radius and neck length as based upon the preceding development.

\section{RESULTS AND DISCUSSION}

Equations (11) and (12) were integrated using initial conditions consistent with the experiments used to obtain the stereological results illustrated in Figures 1 and 2. A starting density of $300 \mathrm{~kg} \mathrm{~m}^{-3}$, coordination number of 2.56 , bond radius $1.0 \times 10^{-4} \mathrm{~m}$, a grain radius of $5.0 \times 10^{-4} \mathrm{~m}$ and a neck length of $0.7 \times 10^{-4} \mathrm{~m}$ were used as starting conditions. The coefficient $A$ in the ice constitutive law was chosen to correspond to that of snow ice rather than columnar ice or pure ice single crystals, since necks contain a relatively large amount of grain boundary and therefore a large number of lattice imperfections in the form of dislocations, vacancies and interstitial atoms. The chosen value of $A$ was $3 \times 10^{-24} \mathrm{~Pa}^{-3} \mathrm{~s}$ (Michel, 1978). The new contact areas formed when grains come into contact with each other during compression were assumed to have radii of $0.33 \times 10^{-4} \mathrm{~m}$, which is the resolution capability of the image-analysis system used earlier (Edens and Brown, 1991). The deformation rate was set at a value of $\mathrm{d} \rho / \mathrm{d} t=1.0 \mathrm{~kg} \mathrm{~m}^{-3} \mathrm{~s}^{-1}$, so that a compression from 300 to $600 \mathrm{~kg} \mathrm{~m}^{-3}$ would be accom-

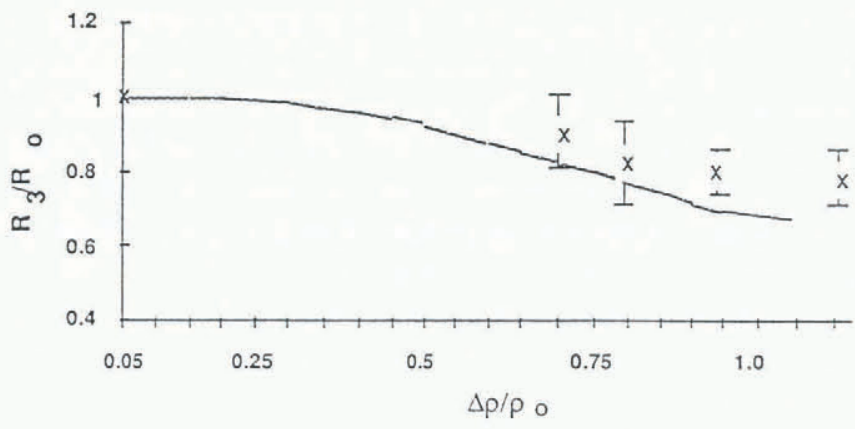

Fig. 6. Comparison of theory with data for variation of three-dimensional bond radius $R_{3}$ with density change $\Delta \rho$. The vertical lines represent data scatter.

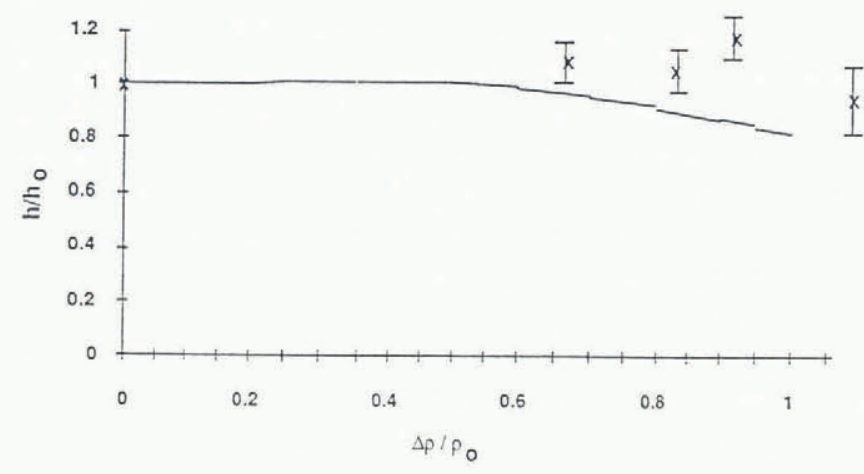

Fig. 7. Comparison of theory with test results for variation of neck length with densification. Vertical lines represent total range of data scatter. The vertical bars represent the total data scatter.

plished in $100 \mathrm{~s}$. This value approximates the deformation rates used in the compression tests. Finally, a value of $C=10$ is used in the expression for the geometric effect. This value was chosen, since investigations of several surface sections indicated that the average slope of the ice surface at the juncture of the grain and neck was much less than the value of $\tan \varphi$ obtained in Equation (2) by using the assumption of spherical grains (Fig. 4).

The initial values of $L, h$ and $R$ were chosen for snow that had been stored for an extended period of time (in excess of 6 months) at $-20^{\circ} \mathrm{C}$. Consequently, it had a fairly large grain-size with significant intergranular bonding and showed very little faceting. Figure 5 illustrates a surface section of snow typical of what was used for this investigation and for the earlier study (Edens and Brown, 1991).

Figures 6 and 7 illustrate the results of the computations. As can be seen in Figure 6, the values of the bond radius computed by Equation (12) decrease uniformly with density. Superimposed on the curve are data from the experiments and stereological analysis. The predicted trends for the bond radius agree fairly well with the data. As indicated earlier, the vertical bars represent the total data scatter. The theoretical curve does underestimate the data values somewhat but the trends are represented.

The data show no consistent trend of neck-length change with volumetric strain, as illustrated in Figure 7. 
There is actually an increase in neck length relative to initial neck length until a value of $\Delta \rho / \rho_{0}=0.9$ is exceeded. However, the theoretical calculations show a very small increase in neck length (up to $5 \%$ ) with volumetric strain until a value of $\Delta \rho / \rho_{0}=0.5$ is reached, after which the neck length begins to decrease. The geometric effect in Equation (11) does not appear to be significant enough to produce a significant positive change in neck length. The variation of bond radius, on the other hand, compares reasonably well with the data.

The question concerning the inability to reproduce the data points for the changes in neck length still remains. Since the variations in bond radius were suitably predicted with the theoretical development, one would expect the theory to be able to determine neck-length changes. The theory, of course has some simplifying assumptions regarding the geometry of both the necks and grains, so one would expect some deviation between theory and data. One answer might lie with the criterion used to determine the neck length in the surface sections analyzed in the earlier study (Edens and Brown, 1991). The neck was defined to begin at the point where the surface curvature changed from convex outward to concave outward as the neck was approached on the surface section from the grain. This is a somewhat subjective criterion which is difficult to use, as the point of curvature change is often difficult to determine. This is especially true when the grain geometry is complicated and highly variable, which is the case with alpine snow. In the case where snow was made from spherical snow particles formed by ejecting water droplets into a liquid nitrogen bath, the neck length would be much more reliably determined, since the particle size and shape was much more uniform. The same could be said for snow that has gone through a melt/freeze process in which the grains are rounded and the neck geometry is very clearly defined.

Another possible answer might be that the imageanalysis system did not have the resolution and screen size for large magnifications to obtain more precise pictures of the neck regions. A system with a higher resolution might eliminate much of the problem. The fact that the data show an erratic pattern suggests that the neck lengths were not being measured reliably.

In view of the above results, a new definition for neck length might be needed, although the one used here is technically sound. Changes in surface-curvature sign do regularly occur and are readily detectable when the grain geometry is simple, as in the case of spherical particles. However, for alpine snow the complicated grain geometry might preclude this criterion as one which is usable. Necks are considered to be an important microstructural parameter which have a significant effect on material properties. More study needs to be conducted to develop better stereological techniques for measuring them.

\section{ACKNOWLEDGEMENTS}

The work reported here was supported by the U.S. Army Research Office (grant No. DAAG29-85-K-0259). The authors wish to express their appreciation of the support by the Geoscience Program in the U.S. Army Research Office.

\section{REFERENCES}

Brown, R.L. 1980. A volumetric constitutive law based on a neck growth model. J. Appl. Phys., 51(1), 161165.

Edens, M. Q. and R.L. Brown. 1991. Changes in microstructure of snow under large deformations. J. Glaciol., 37(126), 193-202.

Gubler, H. 1978a. An alternate statistical interpretation of the strength of snow. J. Glaciol., 20(83), 343-357.

Gubler, H. 1978b. Determination of the mean number of bonds per snow grain and of the dependence of the tensile strength of snow on stereological parameters. J. Glaciol., 20(83), 329-341.

Hansen, A. C. and R. L. Brown. 1986. The granular structure of snow: an internal-state variable approach. J. Glaciol., 32(112), 434-438.

Kry, P.R. 1975a. Quantitative stereological analysis of grain bonds in snow. J. Glaciol., 14(72), 467-477.

Kry, P.R. 1975b. The relationship between the viscoelastic and structural properties of fine-grained snow. J. Glaciol., 14(72), 479-500.

Maeno, N. and T. Ebinuma. 1983. Pressure sintering of ice and its implication to the densification of snow at polar glaciers and ice sheets. J. Phys. Chem., 87(21), 4103-4110.

Michel, B. 1978. Ice mechanics. Québec, Presses de L'Université Laval.

Underwood, E. E. 1970. Quantitative stereology. Reading, MA, Addison-Wesley.

The accuracy of references in the text and in this list is the responsibility of the authors, to whom queries should be addressed. 